\title{
Caractérisation de l'infestation de Tapinanthus dodonaeifolius (Loranthaceae) chez le karité (Vitellaria paradoxa) au Tchad
}

Received: 2019-04-18; revised: 2020-10-20; accepted: 2020-12-08

\author{
Idriss Tourgou Kanika1, Christophe Djekota ${ }^{2}$, Elvire Hortense Biye ${ }^{1}$
}

\author{
${ }^{1 *}$ Université de Yaoundé I, Faculté des Sciences, B.P. 802 , Yaoundé/Cameroun \\ Tél. : (235) 664405 75, E-mail : kanika@yahoo.fr / biye@yahoo.fr \\ ${ }^{2 *}$ Université de N'Djaména, Faculté des Sciences Exactes et Appliquées, Laboratoire de Botanique Systématique et \\ d'Écologie Végétale, B.P. 1027, N’Djaména/Tchad, \\ Tél. : (235) 662831 26, E-mail : cdjekota@yahoo.fr \\ Auteur correspondant Tél.: (235) 662831 26, E-mail :cdjekota@yahoo.fr
}

\begin{abstract}
Résumé: Au Tchad, à cause de ses retombées financières une attention particulière est prêtée aux arbres à karité (Vitellaria paradoxa C.F.Gaertn.). Cependant, cette culture est menacée par les plantes vasculaires parasites de la famille des Loranthaceae. La présente étude a été effectuée dans 3 sites dans la région du Mandoul pour évaluer l'ampleur des attaques de Loranthaceae (gui africain) sur des arbres en fonction des classes de circonférence du tronc à 1,5 $\mathrm{cm}$ du sol. Elle a consisté à dénombrer sur une de surface, les arbres à karité infestés et les touffes de parasites rencontrées sur ces arbres, afin de déterminer leur taux et leur intensité d'infestation. Les résultats obtenus montrent que Tapinanthus dodonaeifolius (DC) Danser a été trouvée comme la seule espèce de Loranthaceae qui parasite les arbres karité étudiés dans la zone d'étude. Le taux moyen d'infestation estimé à $73 \%$ augmente avec l'âge des arbres karité. La moyenne d'intensité de l'infestation/arbre (2,75 touffes à Békôh, 2,27 à Yomi and 2,04 à Bébopen) montre que Tapinanthus dodonaeifolius constitue une réelle menace pour les peuplements de karité dans la zone d'étude. Il reste à rechercher le seuil d'infestation qui provoque une réduction significative de la fructification. Pour l'instant, bien que pénible à cause de la hauteur des arbres adultes, la lutte mécanique contre les Tapinanthus par la coupe systématique des branches infestées est urgente dans les parcs à karité dans cette zone d'étude.
\end{abstract}

Mots clés: Karité, Loranthaceae, parasites Tapinanthus, Tchad.

\section{ChaRACterization of TAPINANTHUS dodonaEIFolius (LoRANTHACEAE) INFESTATION OF SHEA BUTTER TRESS IN CHAD}

Summary: In Chad, special attention is paid to shea trees (Vitellaria paradoxa C.F.Gaertn.) because of their economic importance However, this crop is threatened by parasitic vascular plants of the family Loranthaceae. The present study was conducted at 3 sites in the Mandoul region to assess the extent of Loranthaceae (African mistletoe) attacks on trees based on trunk circumference classes at $1.5 \mathrm{~cm}$ from the ground. It consisted of counting the infested shea trees growing in a certain area and the tufts of parasites found on these trees, in order to determine their rate and intensity of infestation. The results show that Tapinanthus dodonaeifolius (DC) Danser was found as the only species of Loranthaceae that parasitizes shea trees studied in the study area. The average infestation rate was $73 \%$ on average and increased with age of shea trees. The average intensity of infestation per tree (2.75 in Békôh, 2.27 at Yom and 2.04 at Bébopen) shows that Tapinanthus dodonaeifolius represents a real threat for shea stands in the study area. It remains to find the threshold of infestation that causes a significant reduction in fruiting. For the moment, although difficult because of the height of the adult trees, the mechanical fight against the Loranthaceae by the systematic cutting of the infested branches is urgent in the shea parks in this zone of study.

Key words: Chad, Loranthaceae, parasitism, Shea.

\section{Befall des Schibutterbaums duRCh TAPINANTHuS dodonaEIFolius (LORANTHACEAE) IM TCHAD}

Zusammenfassung: Im Tschad wird Schibutterbäumen (Vitellaria paradoxa C.F.Gaertn.) aus ökonomischen Gründen besondere Aufmerksamkeit gewidmet. Da deren Ertrag durch parasitäre Gefäßpflanzen der Familie Loranthaceae (Afrikanische Misteln) verringert wird, wurde an drei Orten der Mandoul-Region eine Bestandsaufnahme des Loranthaceae-Befalls der Bäume in Abhängigkeit der Stammumfangsklassen (gemessen in 1,5 cm Höhe über dem Boden) durchgeführt. Zur Emittlung von Befallsrate und Befallsintensität wurden auf einer bestimmten Fläche die befallenen Sheabäume und die darauf wachsenden Parasitenbüschel gezählt. Die Befallsrate von durchschnittlich 73 \% steigt mit dem Alter der Bäume. Die durchschnittliche Intensität des Befalls pro Baum (2,75 Büschel in Békôh, 2,27 in Yom und 2,04 in Bébopen) zeigt, dass Tapinanthus dodonaeifolius (DC) Danser, die einzige im Untersuchungsbiet auf Schibutterbäumen gefundene Loranthaceae, eine echte Bedrohung für Shea-Bestände darstellt. Zukünftig sollte die Schwelle des Befalls ermttelt werden, die eine signifikante Verringerung der Fruchtbildung bewirkt. Obwohl aufgrund der Höhe der erwachsenen Bäume nicht einfach, ist die mechanische Kontrolle von Tapinanthus durch systematisches Schneiden befallener Äste derzeit dringend erforderlich.

Schlagworte: Chad, Loranthaceae, Parasitismus, Schibutterbaum. 


\section{INTRODUCTION}

$\mathrm{Au}$ Tchad, la zone de peuplement naturelle du karité couvre les sept régions administratives de la zone méridionale du pays (Logone Occidental, Logone Oriental, Mandoul, Mayo Kebbi Est, Mayo Kebbi Ouest, Moyen Chari et Tandjilé). A raison d'une productivité estimée à $15 \mathrm{~kg} / \mathrm{karité} / \mathrm{an}$, les 92683130 pieds de karité dans les 7 régions produiraient 1390247 tonnes de noix par an. Considérant une estimation de $40 \%$ de perte de tous ordres, 834148 tonnes d'amandes/ an sont transformables en beurre (DJEKOTA et al. 2014).

En effet, l'Union Européenne a autorisé l'incorporation des huiles végétales (et notamment le beurre de karité) comme substitut au beurre de cacao dans les chocolats. La proportion de la substitution atteint même déjà $8 \%$ voire $15 \%$ parce que les huiles de substitution reviennent de 10 à $40 \%$ moins cher.

Avec les différents appuis à la filière au Tchad dans cinq dernières années, le karité a acquis une valeur marchande accentuant du coup la concurrence des populations loca-
Longtemps considérés comme sans importance, à cause de la pénibilité d'atteindre les touffes de parasites qui sont en hauteur, l'inquiétude des paysans grandit face à la présence de plus en plus marquée des Loranthaceae est général. C'est pourquoi, il est urgent de mener des études pour mieux orienter la lutte contre ces parasites.

\section{Matériel et MÉthodes}

\subsection{Localisation et caractéristiques de la zone d'étude}

La Région du Mandoul au Sud du Tchad située entre le $9^{\mathrm{e}}$ //35' et $8^{\mathrm{e}} / / 30^{\prime}$ est la zone de cette étude (Fig. 1). 3 sites (Békôh, Yomi et Bébopen) ont été échantillonnés pour servir de cadre à cette étude. Ce choix se justifie par le fait que tous les sites retenus comportent un peuplement représentatif de karité et présentent un gradient écologique nord-sud permettant une meilleure prise en compte des paramètres variables. Les caractéristiques des 3 sites sont présentées sur le Tableau 1.

Tableau 1: Caractéristiques des sites d'études. / Characteristics of study sites.

\begin{tabular}{|l|c|c|c|}
\hline Sites & Békôh & Yomi & Bébopen \\
\hline Latitude & $8380 \mathrm{~N}$ & $8340 \mathrm{~N}$ & $8250 \mathrm{~N}$ \\
\hline Longitude & $17180 \mathrm{E}$ & $17180 \mathrm{E}$ & $17166 \mathrm{E}$ \\
\hline Localisation & $14 \mathrm{~km}$ au NE de Bédjondo & $15 \mathrm{~km} \mathrm{au} \mathrm{SE} \mathrm{de} \mathrm{Bédjondo}$ & $25 \mathrm{~km}$ au S de Bédjondo \\
\hline Type de sol & argilo-limoneux ou sablonneux ferrugineux & sablonneux, latéritique, férralitique \\
\hline Formation végétale & \multicolumn{2}{|c|}{ savane boisée (et maraichage le long des la rivières) } \\
\hline Activités principales & \multicolumn{2}{|c|}{ Agriculture et la pêche, au Yomi + l'apiculture, au Bébopen + la chasse } \\
\hline Pluviométrie & \multicolumn{2}{|c|}{$900-1200 \mathrm{~mm}$} \\
\hline
\end{tabular}

les pour la collecte des fruits. Malheureusement, cet arbre à usages multiples, vital pour les ménages vulnérables, est de plus en plus menacé par les parasites vasculaires appartenant à la famille des Loranthaceae. Les Loranthaceae sont des buissons épiphytoides chlorophylliens qui vivent en hémiparasitismes sur les branches des plantes ligneuses, spontanées ou cultivées. Ces arbustes sont liés à leur hôte par un véritable pont structural et physiologique constitué par un système d'absorption ou suçoir (KuIJT 1969). Le suçoir permet le prélèvement de l'eau et des substances minérales à leur profit (BANNISTER \& GRAHAM STRONG 2002). La dissémination des Loranthaceae est assurée par les oiseaux granivores qui consomment leurs fruits (PRIYA 1983, Boussim 2002). Les fruits des Loranthaceae sont des pseudo-baies (EDOUARD 1989, Boussim et al. 1993). La famille des Loranthaceae est largement répandue dans le monde; elle comprend 77 genres et 950 espèces (PolHILL 1998). Boussim et al. (1993) rapporte qu'au Burkina-Faso, 95\% de la population des karités seraient infestés par quatre espèces de Loranthaceae, à savoir: Tapinanthus dodonaeifolius, Tapinanthus bangwensis, Tapinanthus globiferus, Tapinanthus sophioides. VANDERVEKEN (1993) et HouÉnOn (1997) ont montré que les Loranthaceae affectent la croissance et réduisent la productivité des arbres hôtes qui finissent par mourir. Dibong et al. (2009) a signalé néanmoins l'usage des Loranthaceae en ethno pharmacologie pour le traitement d'au moins 22 maladies au Cameroun.

\subsection{Matériel}

Le matériel végétal est composé des pieds de karité présents dans les parcelles prospectées et leur taux d'infestation par Tapinanthus (Phanérogame parasite de la famille des Loranthaceae).

\subsection{Méthodes}

\subsubsection{Echantillonnage}

Une surface totale de 28 hectares (placettes carrées de 100 $\mathrm{m}$ de coté de chacun), soit 12 hectares à Békoh/Peni, 9 hectares à Bébopen et 7 hectares à Yomi a été étudié à l'aide d'une carte d'occupation du sol de 2013 de la zone d'étude.

Des enquêtes ethnobotaniques auprès de 106 ménages ayant une tranche d'âge moyenne de 60 ans ont été interviewés, soit 59 ménages à Bekôh, 36 à Yomi et 11 à Bébopen (tableau 1). Les ménages exploitants régulièrement ou périodiquement le karité ont été privilégiés dans ces 3 villages.

\subsubsection{Collecte des données}

La collecte des données a été réalisée à partir de transects de $1,5 \mathrm{~km}$ matérialisés dans les terroirs des 3 villages. A l'intérieur de chaque placette, tous les individus de karités (jeunes ou vieux) se trouvant à l'intérieur des placettes de $(100 \mathrm{~m} \times 100 \mathrm{~m})$ délimitées le long des transects ont été examinés. 


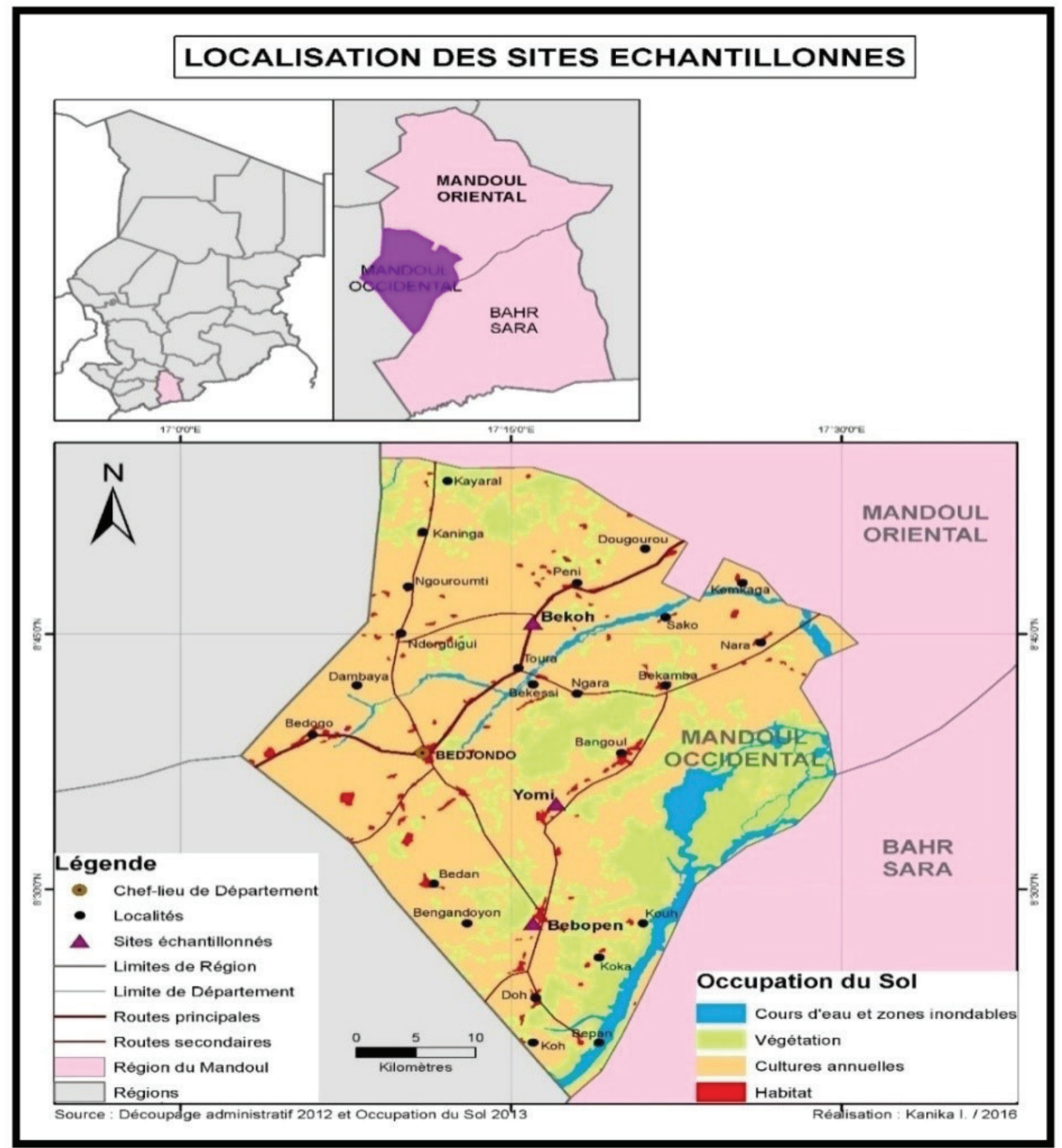

Fig. 1: Localisation de la zone d'étude et des sites échantillonnés. / Location of the study area and sampled sites.

Les individus dont la circonférence à $1,5 \mathrm{~m}$ du sol est (10 $\geq \mathrm{C}<30 \mathrm{~cm}$ ) sont considérés comme des karités jeunes $(<15$ ans $)$ et ceux de $(>30 \mathrm{~cm})$ sont des karités adultes $(>$ 15 ans) (Oyen \& Lemmens 2001). Pour chaque individu recensé, la présence ou non de parasites ainsi que de fruits ont été notée. Des informations complémentaires portant sur le nombre de touffes de Tapinanthus par branches et le nombre de branches saines selon les individus jeunes ou vieux ont aussi été notées. Des échantillons de Tapinanthus ont été prélevés et conservés dans de l'éthanol pour confirmation ultérieure au laboratoire. Les déterminations des espèces ont été faites à l'aide des flores de West Tropical Africa de Hutchinson \& Dalziel (1958) et du Sénégal (Berhaut
1967, 1979). Elles ont été confirmées par la consultation des échantillons des Herbiers du Laboratoire Zootechnie et Vétérinaire/N'Djaména.

Les comptages ont porté sur (i) le nombre de pieds de karité infestés et (ii) le nombre de touffes de parasites présents sur chaque arbre. Ces données ont permis de déterminer:

- le taux d'infestation qui est le pourcentage de plants infestés;

- l'intensité d'infestation exprimée, ici, par le nombre moyen de touffes de Tapinanthus observées par individu. 
La validité des données a été analysée à l'aide du logiciel XLSTAT version 7.5. Ce programme prévoit, en cas de différences significatives entre les moyennes, une comparaison par le test de Newmann - Keuls, pour voir quelles paires de moyennes spécifiques sont différentes.

\section{RÉSULTATS}

\subsection{Loranthaceae rencontrées chez le karité au Tchad}

Cette étude a montré que c'est le parasite $T$. dodonaeifolius (Fig. 2) qui est dominant dans les 3 sites. Selon BousSIM (1991) dans les régions aux conditions climatiques et édaphiques assez favorables, T. dodonaeifolius forme parfois des branches robustes pouvant supporter le poids d'une personne adulte. Dans les régions peu favorables $T$. dodonaeifolius vit en touffes, certes nombreuses mais maigres et rabougries sur des plantes déjà affaiblies par un environnement hostile. En Afrique, T. dodonaeifolius est présente depuis le Sénégal, le Mali, le Tchad jusqu'à la République Démocratique du Congo, la République Centrafricaine et l'Ouganda.

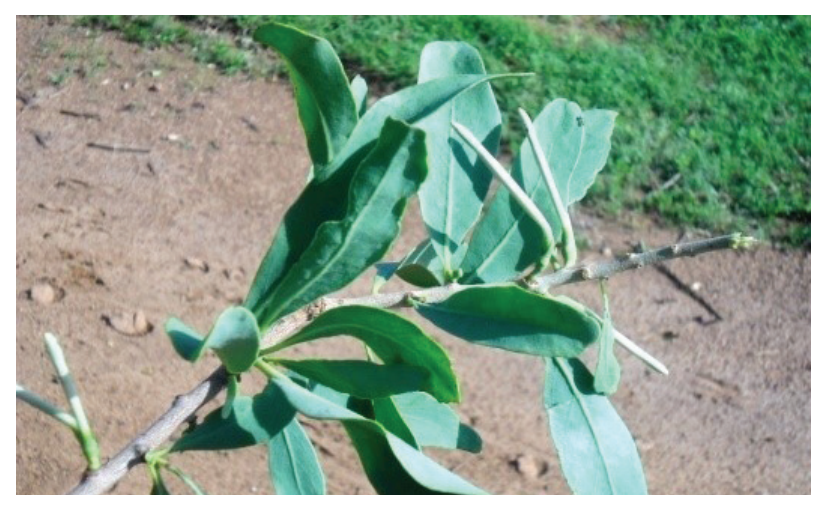

Fig. 2: Rameau florifère de Tapinanthus dodonaeifolius. / Flowering branch of Tapinanthus dodonaeifolius.

Photo: Kanika Idriss, novembre 2107.

\subsection{Caractérisation de l'infestation}

\subsubsection{Taux et intensité d'infestation}

Tapinanthus dodonaeifolius est une espèce de Loranthaceae qui occupe des biotopes héliophiles et se développe généralement à la périphérie du houppier de son hôte de façon à bénéficier au maximum de la lumière solaire.

Dans la zone d'étude, 270 pieds de karités ont été inventoriés dont 244 en âge de produire repartir sur les trois sites, 197 individus portent sur leurs branches plus de deux touffes de parasites, soit un taux d'infestions de $73 \%$. Le nombre des karités parasités par rapport à l'effectif total montre un taux d'infestation croissant allant des jeunes individus aux adultes. Ces résultats montrent que $T$. dodonaeifolius semble avoir une préférence pour les individus adultes.

Le Tableau 2 montre que les taux d'infestation du karité par T. dodonaeifolius augmentent selon la classe de diamètre. Ces résultats montrent que les régénérations des arbres karité ne sont pas infestées. Les infestations sont observées sur les individus de la classe de diamètre; classe $1:(10 \geq \mathrm{C}<$ $30 \mathrm{~cm})$ sont infestés à $23 \%$, ceux de la classe $2:(30<\mathrm{C}<$
$60 \mathrm{~cm})$ à $76 \%$, ceux de la classe $3:(60<\mathrm{C}<90 \mathrm{~cm})$ à $86 \%$ et ceux de la classe 4: (> à $90 \mathrm{~cm})$ sont parasités à $91 \%$. Il apparait que c'est la classe 4: (> à $90 \mathrm{~cm})$ qui constituent le groupe le plus infesté par rapport à la classe 1 : $(10 \geq \mathrm{C}<$ à $30 \mathrm{~cm}$ ) qui est moins infestés. Ce taux d'infestation n'est pas le même dans les trois sites étudiés.

Tableau 2: Taux d'infestation du karité par Tapinanthus dodonaeifolius. / Shea infestation rate by Tapinanthus dodonaeifolius.

\begin{tabular}{|l|c|c|c|}
\hline & Effectif des & \multicolumn{2}{|c|}{ individus parasités } \\
\cline { 3 - 4 } Stade végétatif & individus & nombre & \% \\
\hline Régénération & 11 & 0 & $0 \%$ \\
\hline Classe 1 & 35 & 8 & 22,85 \\
\hline Classe 2 & 75 & 57 & 76 \\
\hline Classe 3 & 73 & 63 & 86,3 \\
\hline Classe 4 & 76 & 69 & 90,78 \\
\hline Total & 270 & 197 & 72,96 \\
\hline
\end{tabular}

En outre, la Figure 3 indique que les intensités d'infestation des arbres karité augmentent avec la classe de diamètre. Les arbres karité de diamètre de la classe 4: ont plus de 3 touffes/arbre parasité, ceux de la classe 3 ont au moins 3 touffes de parasites/arbre infesté, ceux de la classe 2 ont 2 touffes de parasite/arbre infesté et ceux de la classe 1 ont une intensité moyenne d'infestation d'une touffe/arbre parasité. Il apparait que c'est la class, qui compte des individus le plus infesté par rapport à la classe 1 . Le Tableau 3 montre que ce taux d'infestation n'est pas le même dans les trois sites étudiés.

Pour tous ces paramètres évalués (Tab. 3), la comparaison des valeurs minimales d'une part et d'autre part les valeurs maximales entre les sites montre une faible variation. Le nombre de touffes de parasite $T$. dodonaeifolius inventorié suit une corrélation positive d'une classe de diamètre à une autre.

Pour les individus possédant 2 TOUFFEs, le pourcentage des touffes était de $7,7 \%$ à Yomi, suivi de 5,8\% à Békôh et 4,6\% à Bébopen ; ceux avec 3 touffes présentent un pourcentage de $12,25 \%$ à Yomi, suivi de $3,5 \%$ à Békôh et $3,2 \%$ à Bébopen. Et les individus possédant plus de 3 TOUFFES ont un pourcentage de $1,8 \%$ à Bébopen, suivi de $1,7 \%$ à Békôh et $1,6 \%$ à Yomi.

La variation des moyennes est faible dans les 3 sites pour les individus possédant 2 ou 3 touffes du parasite T. dodonaeifolius. Pour les individus qui possèdent plus de 3 touffes, la moyenne des touffes de Békôh $(2,75)$ est supérieure à celle de Yomi $(2,27)$ et de Bébopen $(2,04)$. Il apparait que la moyenne des touffes à Bébopen est légèrement inférieure à celle de Yomi. Ces résultats montrent qu'à Békôh le taux de parasitage est élevé probablement sous l'effet des conditions écologiques et environnementales comparativement à Yomi et Bébopen.

Quant à l'intensité d'infestation, la moyenne des (2,75 touffes/arbre) à Békôh, (2,27 touffes/arbre) à Yomi, (2,04 touffes/arbre) à Bébopen montrent que $T$. dodonaeifolius constitue une menace potentielle pour l'environnement dans les peuplements de karité dans la zone d'étude. 
Tableau 3: Statistiques descriptives du parasite Tapinanthus dodonaeifolius chez l'arbre de karité par site. / Descriptive statistics of the parasite Tapinanthus dodonaeifolius in the shea tree by site.

\begin{tabular}{|c|c|c|c|c|}
\hline Nombre de touffes & Statistique & Békôh & Bébopen & Yomi \\
\hline \multirow{5}{*}{2 touffes } & Moyenne & 0,06 & 0,09 & 0,03 \\
\hline & $\mathrm{CV} \%$ & 5,8 & 4,6 & 7,7 \\
\hline & Ecart-type & 0,345 & 0,414 & 0,23 \\
\hline & Minimum & 0 & 0 & 0 \\
\hline & Maximum & 2 & 2 & 2 \\
\hline \multirow{5}{*}{3 touffes } & Moyenne & 0,23 & 0,27 & 0,02 \\
\hline & $\mathrm{CV} \%$ & 3,5 & 3,2 & 12,25 \\
\hline & Ecart-type & 0,806 & 0,859 & 0,245 \\
\hline & Minimum & 0 & 0 & 0 \\
\hline & Maximum & 3 & 3 & 3 \\
\hline \multirow{6}{*}{$>3$ touffes } & & & & \\
\hline & Moyenne & 2,75 & 2,04 & 2,27 \\
\hline & $\mathrm{CV} \%$ & 1,6 & 1,8 & 1,7 \\
\hline & Ecart-type & 4,349 & 3,61 & 3,804 \\
\hline & Minimum & 0 & 0 & 0 \\
\hline & Maximum & 16 & 12 & 14 \\
\hline
\end{tabular}

Le coefficient de variation $(\mathrm{CV})$ exprimé en pourcentage (\%) est le chiffre qui multiplie le nombre de touffes chez un arbre karité infesté.

The coefficient of variation (CV) expressed as a percentage is the figure that multiplies the number of tufts in an infested shea tree.

\subsubsection{Dégradation des arbres karité par le parasite T. dodonaeifolius (DC) Danser}

L'observation directe sur le terrain présente les arbres karité qui ont presque totalement perdu leur feuillage; ce dernier étant remplacé par les touffes du parasite $T$. dodonaeifolius (DC) Danser. Selon les paysans les arbres densément parasités ne produisent plus normalement. Très peu des paysans interrogés ont une connaissent relative des moyens de lutte dans les trois sites (Fig. 3).

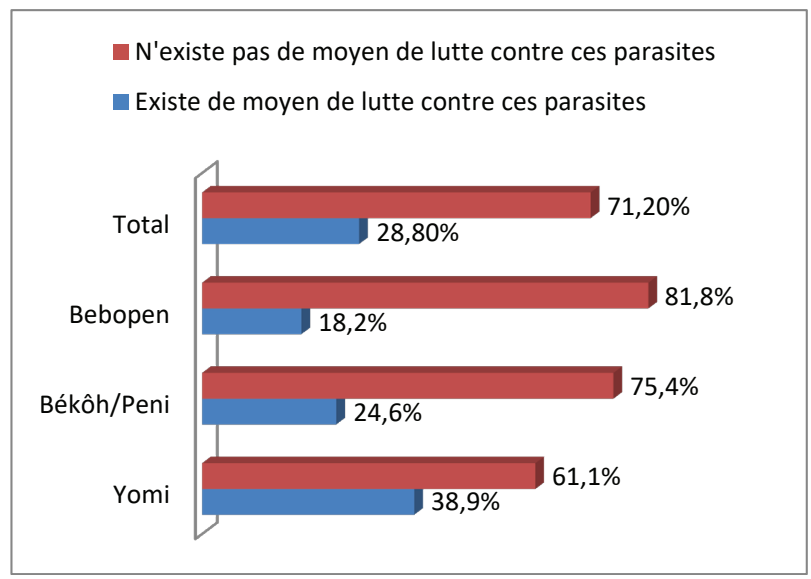

Fig. 3: Existence des moyens de lutte contre les parasites (en tota et selon les sites) / Existence of pest control means (all in all and per site).

Le déparasitage par émondage semble être à la portée des producteurs sauf que les touffes du parasite $T$. dodonaeifolius sont hautes sur les arbres.

\section{Discussion}

Tapinanthus dodonaeifolius (DC) Danser trouvée comme la seule espèce de Loranthaceae qui parasite les arbres karité étudiés dans le Mandoul occidental au Tchad est un résultat qui semble différent de celui de Boussim et al. (1993) qui rapportent qu'au Burkina-Faso, la population des arbres karité sont infestés par quatre espèces de Loranthaceae à savoir: Tapinanthus dodonaeifolius (DC) Danser, Tapinanthus globiferus (A. Rich.) Danser et Tapinanthus aphiodes (Sprague) Danser.

Le taux moyen d'infestation (73\%) obtenu sur ces arbres karité est bas comparativement au taux d'infestation de 95\% obtenus par Boussim et al. (1993) sur les arbres karité au Burkina-Faso]. En outre, le niveau d'infestation qui augmente avec l'âge des arbres karité obtenu dans cette étude a été pareillement montré par ces auteurs.

Quant à l'intensité d'infestation, la moyenne des (2,75 touffes/arbre) à Békôh, (2,27 touffes/arbre) à Yomi, (2,04 touffes/arbre) à Bébopen montrent que $T$. dodonaeifolius constitue une menace les peuplements de karité dans la zone d'étude.

\section{CONCLUSION}

A travers cette étude, il a été indiqué que les arbres karité sont parasités par les Loranthaceae. L'espèce $T$. dodonaeifolius (DC) Danser est apparue comme la plus fréquente sur les arbres karité.

En fonction de l'écologie des 3 sites étudiés, le degré de parasitage des arbres karité étudiés est significativement lié à l'âge des individus. Ainsi, les arbres les plus âgés et ceux 
de longues jachères (jachères de plus de 5 ans étant les plus parasités par les Loranthaceae comparativement aux arbres karité qui sont dans les champs en exploitation.

En perspectives, il serait souhaitable de rechercher les causes de l'apparition des Loranthaceae dans les parcs à karité afin de retarder l'infestation des arbres et l'intensification des Loranthaceae dans les peuplements.

En vue d'adapter les moyens de lutte contre ces parasites les perspectives de recherche orientées sur le type de relation Hôte-parasite qui existe particulièrement entre le karité et l'espèce $T$. dodonaeifolius (DC) Danser permettrait de mieux comprendre et de caractériser cette relation. Il conviendrait également de rechercher l'âge à partir duquel $T$. dodonaeifolius (DC) Danser pourrait avoir un effet significatif sur le rendement des arbres karité. La recherche de clones résistants aux parasites serait également une voie à explorer. Cette étude est à étendre à d'autres régions qui produisent le karité au Tchad.

\section{REMERCIEMENTS}

Nous remercions vivement l'Université de Yaoundé I/Cameroun, Faculté des Sciences qui a encadré ces travaux de recherche. Au Tchad, nous remercions l'Université de N'Djaména, Faculté des Sciences Exactes et Appliquées, Laboratoire de Botanique Systématique et d'Écologie Végétale ensuite les autorités décentralisées du Ministère de l'Intérieur et de l'Environnement de la Région du Mandoul qui nous ont apporté le soutien nécessaire lors de la collecte des données. Aux référés anonymes qui ont lu avec beaucoup d'attention et fait des commentaires très utiles au manuscrit, nous adressons nos sincères gratitudes.

\section{REFERENCES}

Bannister P, Graham Strong L \& Inge A (2002): Differential accumulation of nutrient elements in some New Zealand mistletoes and their hosts. "https://fr.wikipedia.org/ wiki/Commonwealth_Scientific_and_Industrial_Research Organisation".

Berhaut J (1967): Flore du Sénégal. - Clairafrique, 2e éd. Dakar, Sénégal, 485 p.

Berhaut J (1979): Flore illustrée du Sénégal. Tome VI. Ed. Gouvernement du Sénégal. - Clairafrique, Dakar, 403-496.

Boussim I J, SAllé G \& Guinko S (1993): Tapinanthus parasite du karité au Burkina Faso. 1er partie: Identification et distribution. - Bois et Forêt des Tropiques 238: 45-52.

Boussim I J (1991): Contribution à l'étude des Tapinanthus parasites du karité au Burkina Faso. Thèse de doctorat de 3e cycle, Université de Ouagadougou, 152 P.
Boussim I J (2002): Les Phanérogames parasites du Burkina Faso : inventaire, taxonomie, écologie et quelques aspects de leur biologie. Cas particulier des Loranthaceae parasites du karité, Thèse d'État, Université de Ouagadougou,306 p.

Condaminé M (1988): Les Tapinanthus, parasites du karité: prospection au Mali et au Burkina Faso et premières observations histo-cytologiques de la baie. DEA. Université P. et M. Curie (Paris VI), 43 p, non publié.

DAlziel J M (1955): The useful plants of West Africa, appendix of flora of West Africa.

Dibong S D, Engone O L, Ndongo D, Priso R J, TaFfouo V, Fankem H, Salle G, Missoup A D, Boussim I J \& Amougou A (2009): Faut-il éradiquer les Loranthaceae sur les ligneux à fruits commercialisés de la région littorale du Cameroun ? Int. J. Biol. Chem. Sci. 4(3): 555-562. ISSN 1991-8631. http://www.ajol.info/index.php/ijbcs.

Djekota C, Mouga M, Duimramadi A, Duelasem B, Mbayngone E, Maiga R D, Rimgoto K, \& Noubady D (2014): Potentiel karité au Tchad (Vitellaria paradoxa C.F. Gaertn subsp paradoxa, Sapotaceae). J Animal Plant Sci 2071-2074.

Hutchinson J \& DALziel J M (1958): Flora of West Tropical Africa. 2nd edit., Vol.I, Part. 2. Millbank, London, 276 p.

EdOUARD J A (1989): Les phanérogames parasites de la Martinique. Écologie et quelques aspects de leur biologie. Thèse de Doctorat de l'Université de Pierre et Marie Curie, Paris, $305 \mathrm{p}$.

HouÉNON G J (1997): Rapport d'une mission de recherche sur les parasites vasculaires de la famille des Loranthaceae, dans la Sous-Préfecture de Bassila. - CPU/UNB-BENIN, 5 p.

KuiJT J L (1969): The Biology of Parasitic Flowering Plants, University of California Press, Berkeley, Los Angeles, $246 \mathrm{p}$.

Oyen L P A \& Lemmens R H M J (2001): Ressources végétales de l'Afrique tropicale. - Précurseurs: 107-172.

Polhill R \& Wiens D (1998): Mistletoes of Africa. - The Royal Botanic Garden, Kew, 370 p.

PRIYA D (1983): Birds and Neotropical mistletoes: effects on seedling recruitment. - Oecol 60: 271-273.

Sallé G, Boussim I J \& Raynal-Roques A (1991): Le Karité, une richesse potentielle. Perspectives de recherche pour améliorer sa production. Bois Forêts tropiques. - 228 : 1123.

VANDERVEKen J (1993): Les Phanérogames phytopathogènes. - Traité de pathologie végétale, 234-248. 